TRANSACTIONS OF THE

AMERICAN MATHEMATICAL SOCIETY

Volume 350, Number 7, July 1998, Pages 2813-2832

S 0002-9947(98)02198-9

\title{
THE CASTELNUOVO REGULARITY OF THE REES ALGEBRA AND THE ASSOCIATED GRADED RING
}

\author{
NGÔ VIÊT TRUNG
}

Dedicated to the memory of Professor Hideyuki Matsumura

\begin{abstract}
It is shown that there is a close relationship between the invariants characterizing the homogeneous vanishing of the local cohomology and the Koszul homology of the Rees algebra and the associated graded ring of an ideal. From this it follows that these graded rings share the same Castelnuovo regularity and the same relation type. The main result of this paper is however a simple characterization of the Castenuovo regularity of these graded rings in terms of any reduction of the ideal. This characterization brings new insights into the theory of $d$-sequences.
\end{abstract}

\section{INTRODUCTION}

Let $S=\bigoplus_{n \geq 0} S_{n}$ be a finitely generated standard graded ring over a noetherian commutative ring $S_{0}$. For any graded $S$-module $M$ we denote by $M_{n}$ the homogeneous part of degree $n$ of $M$, and we define

$$
a(M):= \begin{cases}\max \left\{n \mid M_{n} \neq 0\right\} & \text { if } M \neq 0, \\ -\infty & \text { if } M=0 .\end{cases}
$$

Let $S_{+}$be the ideal generated by the homogeneous elements of positive degree of $S$. For $i \geq 0$, set

$$
a_{i}(S):=a\left(H_{S_{+}}^{i}(S)\right),
$$

where $H_{S_{+}}^{i}$ (.) denotes the $i$ th local cohomology functor with respect to the ideal $S_{+}$.

The Castelnuovo regularity of $S$ is defined as the number

$$
\operatorname{reg} S:=\max \left\{a_{i}(S)+i \mid i \geq 0\right\}
$$

This is an important invariant of the graded ring $S$ [3] [19]. For instance, if $S$ is represented as a factor ring $S_{0}[T] / \Im$ of a polynomial ring corresponding to a minimal generating set of $S_{1}$, then reg $S$ provides upper bounds for the syzygies of $S$ as an $S_{0}[T]$-module. In particular,

$$
\text { reltype } S \leq \operatorname{reg} S+1 \text {, }
$$

Received by the editors June 15, 1996.

1991 Mathematics Subject Classification. Primary 13A30; Secondary 13D45.

Key words and phrases. Rees algebra, associated graded ring, Castelnuovo regularity, relation type, reduction number, $d$-sequence, filter-regular sequence.

This work is partially supported by the National Basic Research Program of Vietnam.

(C) 1998 American Mathematical Society 
where reltype $S$ denotes the maximum degree of the forms of a minimal basis for $\Im$ with reltype $S=-\infty$ if $\Im=0$. It is well-known that this degree does not depend on the choice of the representation of $S$, and we will call it the relation type of $S$.

Let $A$ be a noetherian commutative ring and $I$ an ideal of $A$. We denote by $R_{I}(A)$ the Rees algebra $\bigoplus_{n \geq 0} I^{n}$ and by $G_{I}(A)$ the associated graded ring $\bigoplus_{n \geq 0} I^{n} / I^{n+1}$ of $A$ with respect to $I$. The aim of this paper is to find a simple characterization of $\operatorname{reg} R_{I}(A)$ and $\operatorname{reg} G_{I}(A)$.

First, we will shows that there is a close relationship between the invariants $a_{i}\left(R_{I}(A)\right)$ and $a_{i}\left(G_{I}(A)\right)$ (Theorem 3.1) from which we can easily derive the formula

$$
\operatorname{reg} R_{I}(A)=\operatorname{reg} G_{I}(A)
$$

of Ooishi [20]. This formula was recently rediscovered by Johnson and Ulrich [15]. There is also a close relationship between the invariants characterizing the homogeneous vanishing of the Koszul homology of $R_{I}(A)$ and $G_{I}(A)$ (Proposition 3.4) which generalizes the formula

$$
\text { reltype } R_{I}(A)=\operatorname{reltype} G_{I}(A)
$$

in the case when $I$ is not generated by a regular sequence. This formula was recently discovered by Planas-Vilanova [21].

Next, we will give characterizations of $\operatorname{reg} R_{I}(A)$ and $\operatorname{reg} G_{I}(A)$ in terms of any reduction of $I$ (Proposition 4.2 and Theorem 4.8). These characterizations are based on a careful analysis of properties of filter-regular sequences of degree 1 in $R_{I}(A)$ and $G_{I}(A)$. The main result is the following.

Theorem 1.1. Let $A$ be a local ring with infinite residue field and I an ideal of $A$. For a given integer $r \geq 0$ and any reduction $J$ of $I$, the following conditions are equivalent:

(i) $\operatorname{reg} R_{I}(A)=r$.

(ii) $\operatorname{reg} G_{I}(A)=r$.

(iii) There is a minimal basis $x_{1}, \ldots, x_{s}$ for $J$ such that

$$
\left[\left(x_{1}, \ldots, x_{i-1}\right): x_{i}\right] \cap I^{r+1}=\left(x_{1}, \ldots, x_{i-1}\right) I^{r}, i=1, \ldots, s,
$$

and $r$ is the least integer $\geq r_{J}(I)$ with this property.

Recall that an ideal $J \subseteq I$ is a reduction of $I$ if $I^{n+1}=J I^{n}$ for some integer $n \geq 0$. The least number $n$ with this property is called the reduction number $r_{J}(I)$ of $I$ with respect to $J$.

Theorem 1.1 sheds new light on the theory of $d$-sequences, which, since its introduction by Huneke [11], [14], has established itself as an important tool for commutative algebra. It is known [6] that a sequence $x_{1}, \ldots, x_{s}$ of elements of $A$ is a $d$-sequence if and only if $x_{1}, \ldots, x_{s}$ is a minimal basis for the ideal $I=\left(x_{1}, \ldots, x_{s}\right)$ and

$$
\left[\left(x_{1}, \ldots, x_{i-1}\right): x_{i}\right] \cap I=\left(x_{1}, \ldots, x_{i-1}\right), \quad i=1, \ldots, s .
$$

Thus, Theorem 1.1 implies the following interesting relation between $d$-sequences and the Castelnuovo regularity, which is implicitly contained in earlier works of Herzog, Simis, and Vasconcelos [7] and Kühl [17].

Corollary 1.2. Let $A$ be a local ring with infinite residue field and $I$ an ideal of $A$. Then $\operatorname{reg} R_{I}(A)=0$ (or, equivalently, $\operatorname{reg} G_{I}(A)=0$ ) if and only if $I$ is generated by a d-sequence. 
Perhaps, the most important property of an ideal generated by a $d$-sequence is that its symmetric algebra is isomorphic to its Rees algebra (see Huneke [11] and Valla [28]) or, in other words, it is of linear type. Recall that an ideal $I$ is called of type $r$ if reltype $R_{I}(A)=r$. Since reltype $R_{I}(A) \leq \operatorname{reg} R_{I}(A)+1$, Corollary 1.2 clearly sets apart the class of ideals generated by $d$-sequences from those of linear type.

We will call a sequence $x_{1}, \ldots, x_{s}$ of elements of a ring $A$ a sequence of regular type $r$ if

$$
\left[\left(x_{1}, \ldots, x_{i}\right): x_{i+1}\right] \cap I^{r+1}=\left(x_{1}, \ldots, x_{i}\right) I^{r}, \quad i=0, \ldots, s-1,
$$

where $I=\left(x_{1}, \ldots, x_{s}\right)$, and $r$ is the least integer with this property. This notion is justified by the following result:

Theorem 1.3. Let $I$ be an arbitrary ideal of a ring $A$. Then:

(i) $\operatorname{reg} R_{I}(A)=\operatorname{reg} G_{I}(A)=r$ if $I$ is generated by a sequence of regular type $r$.

(ii) $I$ is generated by a sequence of regular type $r$ if $A$ is a local ring with infinite residue field and $\operatorname{reg} R_{I}(A)=r$ or $\operatorname{reg} G_{I}(A)=r$.

Sequences of regular type enjoy some interesting properties which cover those of $d$-sequences. In some cases, these properties also bring new insight into the theory of $d$-sequences. For instance, Huneke [12] calls a standard graded ring $S$ a special graded ring if $S_{+}$is generated by a $d$-sequence. He proved that if $I$ is generated by a $d$-sequences, then $R_{I}(A)$ and $G_{I}(A)$ are special graded. Conversely, Kühl [17] showed that if $A$ is a local ring and $G_{I}(A)$ is special graded, then $I$ is generated by a $d$-sequence. We shall see that if $A$ is a local ring and $R_{I}(A)$ is special graded, then $I$ is generated by a $d$-sequence, too, and that similar results also hold for ideals generated by a sequence of any regular type.

This paper grew out of the author's attempt to understand some recent results of Marley and Huckaba. Marley [18] has given some constraints for the local cohomology of associated graded rings of Cohen-Macaulay local rings. We shall see that these constraints are natural consequences of the relationship between $a_{i}\left(R_{I}(A)\right)$ and $a_{i}\left(G_{I}(A)\right)$. Huckaba [9] [10] has proved the bound reltype $R_{I}(A) \leq r_{J}(I)+1$ for several classes of ideals $I$, where $r_{J}(I)$ is the reduction number of a minimal reduction $J$ of $I$. Our idea is to find a similar bound for $\operatorname{reg} R_{I}(A)$. By [24] there is a characterization of the Castelnuovo regularity of an arbitrary standard graded ring $S$ by means of a minimal reduction of $S_{+}$. Translating this characterization for $R_{I}(A)$ and $G_{I}(A)$, we are led to the condition $(\star)$ with all the consequences mentioned above. As an application, we will show that $\operatorname{reg} R_{I}(A)=r_{J}(I)$ for the classes of ideals considered by Huckaba.

It can easily be shown that the condition $(\star)$ can be replaced by the condition

$$
\left[\left(x_{1}, \ldots, x_{i-1}\right) I^{r}: x_{i}\right] \cap I^{r+1}=\left(x_{1}, \ldots, x_{i-1}\right) I^{r}, \quad i=0, \ldots, s .
$$

In the case $\left(x_{1}, \ldots, x_{s}\right)=I$, this condition has already been studied by Costa [2] $(r=0)$ and Raghavan [22] ( $r$ arbitrary). They have shown that if the above condition is satisfied, then reltype $R_{I}(A) \leq r+1$. But this is only a consequence of the bound $\operatorname{reg} R_{I}(A) \leq r$ deduced from Theorem 1.1.

Moreover, the condition $(\star)$ can be used to give a characterization of superficial sequences which does not depend on the asymptotic behavior of the elements (Proposition 6.3) and to study the problem when Proj $R_{I}(A)$ is a Cohen-Macaulay scheme (Theorem 6.5). Notably, we obtain the following result. 
Corollary 1.4. Let $A$ be a local ring which is a homomorphic image of a regular local ring with algebraically closed residue field. Then $\operatorname{Proj} R_{I}(A)$ is a CohenMacaulay scheme for all parameter ideals $I$ of $A$ if and only if every system of parameters of $A$ is a sequence of regular type.

The paper is divided into 6 sections. In Section 2 we recall some definitions and prepare some results related to the Castelnuovo regularity of a graded ring. In Section 3 we will establish the relationships between (co)homological invariants of the associated graded ring and the Rees algebra. In Section 4 we characterize homogeneous filter-regular sequences of degree 1 and thereby the Castelnuovo regularity of these graded rings. Based on these characterizations, in Section 5 we introduce and study the notion of a sequence of regular type. In Section 6 we give the applications mentioned above.

All rings in this paper are assumed to be noetherian commutative rings with unity.

Acknowledgement. It is a pleasure to thank J. Herzog and L.T. Hoa for helpful conversations during the preparation of this paper.

\section{The Castelnuovo Regularity of a graded Ring}

Let $z_{1}, \ldots, z_{s}$ be a sequence of homogeneous elements of a standard graded ring $S$. We call $z_{1}, \ldots, z_{s}$ a filter-regular sequence (with respect to $S_{+}$) if $z_{i} \notin P$ for any associated prime ideal $P$ of $\left(z_{1}, \ldots, z_{i-1}\right), P \nsupseteq S_{+}, i=1, \ldots, s$. Define

$$
a(\mathbf{z}):=\max \left\{a\left(\left(z_{1}, \ldots, z_{i-1}\right): z_{i} /\left(z_{1}, \ldots, z_{i-1}\right)\right) \mid i=1, \ldots, s\right\} .
$$

By [24, Lemma 2.1], $\mathbf{z}$ is a filter-regular sequence if and only if $a(\mathbf{z})<\infty$. We shall often use this characterization of a filter-regular sequence without further mention.

Lemma 2.1 [24, Lemma 2.3]. Let $z$ be a homogeneous filter-regular element of degree 1 of $S$. For all $i \geq 0$,

$$
a_{i+1}(S)+1 \leq a_{i}(S / z S) \leq \max \left\{a_{i}(S), a_{i+1}(S)+1\right\} .
$$

It is assumed in [24] that $S_{0}$ is a local ring. But it is clear that the results there hold for an arbitrary ring $S_{0}$. Notice that the values of $a_{i}(S)$ and $a(\mathbf{z})$ defined here are less than those of [24] by 1 .

Proposition 2.2. Let $\mathbf{z}=z_{1}, \ldots, z_{s}$ be a filter-regular sequence of homogeneous elements of degree 1 of $S$. Then:

(i) $\max \left\{a_{i}(S)+i \mid i=0, \ldots, s-1\right\}=a(\mathbf{z})$ [24, Proposition 2.2].

(ii) $\max \left\{a_{i}(S)+i \mid i=0, \ldots, s\right\}=$

$$
\max \left\{a\left(\left(z_{1}, \ldots, z_{i}\right): S_{+} /\left(z_{1}, \ldots, z_{i}\right)\right) \mid i=0, \ldots, s\right\} .
$$

Proof. We will prove only (ii). Applying Lemma 2.1 successively, we get

$$
a_{i}(S)+i \leq a_{0}\left(S /\left(z_{1}, \ldots, z_{i}\right)\right) \leq \max \left\{a_{j}(S)+j \mid j=0, \ldots, i\right\} .
$$

From this it follows that for $t \leq s$

$$
\max \left\{a_{i}(S)+i \mid i=0, \ldots, t\right\}=\max \left\{a_{0}\left(S /\left(z_{1}, \ldots, z_{i}\right)\right) \mid i=0, \ldots, t\right\} .
$$

Set $a=a_{0}\left(S /\left(z_{1}, \ldots, z_{i}\right)\right)$. If we identify the module $H_{S_{+}}^{0}\left(S /\left(z_{1}, \ldots, z_{i}\right)\right)$ with the ideal $\bigcup_{n \geq 0}\left(z_{1}, \ldots, z_{i}\right): S_{+}^{n} /\left(z_{1}, \ldots, z_{i}\right)$, we have

$$
H_{S_{+}}^{0}\left(S /\left(z_{1}, \ldots, z_{i}\right)\right)_{a} \subseteq\left(z_{1}, \ldots, z_{i}\right): S_{+} /\left(z_{1}, \ldots, z_{i}\right) \subseteq H_{S_{+}}^{0}\left(S /\left(z_{1}, \ldots, z_{i}\right)\right) .
$$


Therefore, $a\left(\left(z_{1}, \ldots, z_{i}\right): S_{+} /\left(z_{1}, \ldots, z_{i}\right)\right)=a$, and the conclusion follows.

Corollary 2.3. Let $s=$ grade $S_{+}$. Then

(i) $a_{i}(S)=-\infty$ for $i<s$,

(ii) $a_{s}(S) \geq-s$,

(iii) $a_{1}(S) \geq-1$ if $H_{S_{+}}^{1}(S) \neq 0$.

Proof. It is clear that $a_{i}(S)$ is the maximum of $a_{i}\left(S_{P}\right)$ over all graded prime ideals $P$ of $S$ for any $i \geq 0$. Therefore, we may restrict to the case when $S_{0}$ is a local ring. Without restriction we may assume that the residue field of $S_{0}$ is infinite. Using prime avoidance we can find a regular sequence $z_{1}, \ldots, z_{s}$ of $s$ homogeneous elements of degree 1 of $S$. Since $\left(z_{1}, \ldots, z_{i}\right): S_{+}=\left(z_{1}, \ldots, z_{i}\right)$ for $i=1, \ldots, s$, Proposition 2.2 (ii) implies that $\max \left\{a_{j}(S)+j \mid j=1, \ldots, i-1\right\}=-\infty$. Hence $a_{i}(S)=-\infty$ for $i=0, \ldots, s-1$. As a consequence,

$$
\begin{aligned}
a_{s}(S)+s & =\max \left\{a_{i}(S)+i \mid i=0, \ldots, s\right\} \\
& =a\left(\left(z_{1}, \ldots, z_{s}\right): S_{+} /\left(z_{1}, \ldots, z_{s}\right)\right) \geq 0 .
\end{aligned}
$$

Therefore, $a_{s}(S) \geq-s$. So we have proved (i) and (ii). To prove (iii) we set $\bar{S}=S / H_{S_{+}}^{0}(S)$. It is easily seen that grade $\bar{S}_{+} \geq 1$ and $H_{\bar{S}_{+}}^{1}\left(\bar{S}_{+}\right) \simeq H_{S_{+}}^{1}(S) \neq 0$. Therefore, $a_{1}(S)=a_{1}(\bar{S}) \geq-1$ by (ii).

By [24, Corollary 3.3], reg $S$ can be characterized in terms of a minimal reduction of $S_{+}$which is generated by a filter-regular sequence of homogeneous elements of degree 1. Now we will extend this result as follows.

Theorem 2.4. Let $\mathbf{z}$ be a filter-regular sequence of homogeneous elements of $S$ of degree 1 which generates a reduction for $S_{+}$. Then

$$
\operatorname{reg} S=\max \left\{a(\mathbf{z}), r_{(\mathbf{z})}\left(S_{+}\right)\right\} .
$$

Proof. Let $\mathbf{z}=z_{1}, \ldots, z_{s}$. By Proposition 2.2 we have

$$
a(\mathbf{z})=\max \left\{a\left(\left(z_{1}, \ldots, z_{i}\right): S^{+} /\left(z_{1}, \ldots, z_{i}\right)\right) \mid i=0, \ldots, s-1\right\} .
$$

Further, it is easy to see that

$$
\begin{aligned}
r_{(\mathbf{z})}\left(S_{+}\right) & =\max \left\{n \mid(\mathbf{z})_{n} \neq S_{n}\right\}=a(S /(\mathbf{z})) \\
& =a\left(\left(z_{1}, \ldots, z_{s}\right): S_{+} /\left(z_{1}, \ldots, z_{s}\right)\right) .
\end{aligned}
$$

Therefore,

$$
\begin{aligned}
\max \left\{a(\mathbf{z}), r_{(\mathbf{z})}\left(S_{+}\right)\right\} & =\max \left\{a\left(\left(z_{1}, \ldots, z_{i}\right): S^{+} /\left(z_{1}, \ldots, z_{i}\right)\right) \mid i=0, \ldots, s\right\} \\
& =\max \left\{a_{i}(S)+i \mid i=0, \ldots, s\right\} .
\end{aligned}
$$

It remains to show that $H_{S_{+}}^{i}(S)=0$ for $i>s$. If $s=0$, we have $S_{n}=0$ for all large $n$, hence $H_{S_{+}}^{i}(S)=0$ for $i>0$. If $s \geq 1$, using induction we may assume that $H_{S_{+}}^{i}\left(S / z_{1} S\right)=0$ for $i>s-1$. By Lemma 2.1, this implies $H_{S_{+}}^{i}(S)=0$ for $i>s$. The proof is now complete.

Remark. If $S_{0}$ is a local ring, there is a similar relationship between a minimal reduction of $S_{+}$and the invariants $a_{i}^{*}(S):=a\left(H_{M}^{i}(S)\right)$, which characterize the homogeneous vanishing of the local cohomology modules of $S$ with respect to the maximal graded ideal $M$ ([1, Proposition 2.7]; [25, Theorem 2.2]). 
With regard to Theorem 2.4 one may ask when a reduction of $S_{+}$can be minimally generated by a filter-regular sequence of homogeneous elements of degree 1 . For this we already have the following answer.

Lemma 2.5 [24, Proof of Lemma 3.1]. Let $S$ be a standard graded ring over a local ring $S_{0}$ with infinite residue field. Then any reduction of $S_{+}$generated by forms of degree 1 is minimally generated by a filter-regular sequence of homogeneous elements of degree 1 .

Remark. If $S_{0}$ is a local ring with maximal ideal $\mathfrak{n}$, we define the analytic spread of $S_{+}$by $\ell\left(S_{+}\right):=\operatorname{dim} S / \mathfrak{n} S$. It is known that any minimal reduction for $S_{+}$is minimally generated by $\ell\left(S_{+}\right)$elements and that $\max \left\{i \mid H_{S_{+}}^{i}(S) \neq 0\right\}=\ell\left(S_{+}\right)$.

There is also a characterization of the Castelnuovo regularity and the relation type of a graded ring by means of Koszul homology. This result was originally formulated for a standard graded ring $S$ over a local ring $S_{0}$, but the proof works for any ring $S_{0}$.

Proposition $2.6\left[23,(2.2)\right.$ and (4.1)]. Let $Q$ be any reduction of $S_{+}$. Let $H_{i}(Q, S)$ denote the ith homology module of the Koszul complex of $S$ with respect to $Q$. Then

$$
\operatorname{reg} S=\max \left\{a\left(H_{i}(Q, S)\right)-i \mid i \geq 0\right\} .
$$

Furthermore,

$$
\text { reltype } S=a\left(H_{1}\left(S_{+}, S\right)\right)
$$

\section{The Relationships Between the Rees Algebra AND THE ASSOCIATED GRADED RING}

Let $A$ be a ring and $I$ an ideal of $A$. For brevity we shall denote $R_{I}(A)$ by $R$ and $G_{I}(A)$ by $G$. We shall often consider $R$ as the subring $A[I t]$ of the polynomial ring $A[t]$ and $G$ as the quotient ring $R / I R$.

Our approach will be based on the following exact sequences:

$$
\begin{gathered}
0 \rightarrow R_{+} \rightarrow R \rightarrow A \rightarrow 0, \\
0 \rightarrow R_{+}(1) \rightarrow R \rightarrow G \rightarrow 0,
\end{gathered}
$$

where $A$ is considered as a graded ring concentrated in degree zero. This approach has been used recently by Johnson and Ulrich in order to prove that $\operatorname{reg}(R)=$ $\operatorname{reg}(G)$ [15, Proposition 4.1].

Theorem 3.1. Let $I$ be an ideal of a ring $A$. Then

(i) $a_{i}(R) \leq a_{i}(G), i \neq 1$.

(ii) $a_{i}(R)=a_{i}(G)$ if $a_{i}(G) \geq a_{i+1}(G), i \neq 1$.

(iii) The statements (i) and (ii) hold for $i=1$ if $H_{G_{+}}^{1}(G) \neq 0$ or if $I \subseteq \sqrt{0}$.

(iv) $a_{1}(R)=-1$ if $H_{G_{+}}^{1}(G)=0$ and $I \nsubseteq \sqrt{0}$.

Proof. For short, set $H^{i}()=.H_{R_{+}}^{i}($.$) . Since H^{0}(A)_{n}=0$ for $n \neq 0$ and $H^{i}(A)=0$ for $i \geq 1$, from the exact sequence (1) we can derive that $H^{i}\left(R_{+}\right)_{n} \simeq H^{i}(R)_{n}$ for $n=0, i \geq 2$, and for $n \neq 0, i \geq 0$. Since $H_{G_{+}}^{i}(G)=H^{i}(G)$, (2) induces the exact sequence

$$
H^{i}\left(R_{+}\right)_{n+1} \rightarrow H^{i}(R)_{n} \rightarrow H^{i}(G)_{n} \rightarrow H^{i+1}\left(R_{+}\right)_{n+1} .
$$


Replacing $H^{i}\left(R_{+}\right)_{n+1}$ by $H^{i}(R)_{n+1}$ and setting $H^{i}(G)_{n}=0$ whenever that is possible, we get a surjective map $H^{i}(R)_{n+1} \rightarrow H^{i}(R)_{n}$ for $n \geq \max \left\{0, a_{i}(G)+1\right\}$ if $i=0,1$ and for $n \geq a_{i}(G)+1$ if $i \geq 2$. Since $H^{i}(R)_{n}=0$ for all large $n$, it can be successively deduced that

$$
\begin{gathered}
H^{i}(R)_{n}=0 \text { for } n \geq \max \left\{0, a_{i}(G)+1\right\} \text { if } i=0,1 \\
\text { and for } n \geq a_{i}(G)+1 \text { if } i \geq 2 .
\end{gathered}
$$

As an immediate consequence, $a_{i}(R) \leq a_{i}(G)$ for $i \geq 2$. For $i=0$ we have to distinguish two cases. If $H^{0}(G)=0, a_{0}(G)=-\infty$. Hence $H^{0}(R)_{n}=0$ for all $n \geq 0$ by (3). From this it follows that $H^{0}(R)=0$. Therefore, $a_{0}(R)=-\infty=a_{0}(G)$. If $H^{0}(G) \neq 0, a_{0}(G) \geq 0$. Hence $H^{0}(R)_{n}=0$ for $n \geq a_{0}(G)+1$ by (3), which implies $a_{0}(R) \leq a_{0}(G)$. So we obtain (i).

If $H^{1}(G) \neq 0, a_{1}(G) \geq-1$ by Corollary 2.3 (iii). Hence $H^{1}(R)_{n}=0$ for $n \geq a_{1}(G)$ by (3), which implies $a_{1}(R) \leq a_{1}(G)$. If $I \subseteq \sqrt{0}$, then $H^{i}(R)=0$ and $H^{i}(G)=0$ for all $i \geq 1$. Hence $a_{1}(R)=a_{1}(G)=-\infty$. So we have proved the first part of (iii).

Now we will prove (ii) and the second part of (iii). It is sufficient to show that $a_{i}(R) \geq a_{i}(G)$. For this we may assume that $a_{i}(G) \neq-\infty$. For $i=0$, we have either $a_{1}(R) \leq-1$ or $a_{1}(R) \leq a_{1}(G)$ by $(3)$. For $i \geq 1$, we have $a_{i+1}(G) \geq a_{i+1}(R)$ by (i). Hence the assumption $a_{i+1}(G) \leq a_{i}(G)$ implies $a_{i+1}(R) \leq a_{i}(G)$. Set $n=a_{i}(G)$. Then $H^{i+1}\left(R_{+}\right)_{n+1} \simeq H^{i+1}(R)_{n+1}=0$. Putting this into the above exact sequence, we get a surjective map $H^{i}(R)_{n} \rightarrow H^{i}(G)_{n}$. Since $H^{i}(G)_{n} \neq 0$, $H^{i}(R)_{n} \neq 0$. Therefore, $a_{i}(R) \geq a_{i}(G)$.

To prove (iv) we assume that $H^{1}(G)=0$. Then $a_{1}(G)=-\infty$. Hence $a_{1}(R) \leq-1$ by (3). If $a_{1}(R)<-1, H^{1}(R)_{-1}=0$. Since $H^{0}(G)_{-1}=0$, from (2) we can deduce that $H^{1}\left(R_{+}\right)_{0}=0$. Now, using (1) we get the exact sequence

$$
H^{0}\left(R_{+}\right)_{0} \rightarrow H^{0}(R)_{0} \rightarrow H^{0}(A) \rightarrow 0
$$

But $H^{0}\left(R_{+}\right)_{0}=0$ because $\left(R_{+}\right)_{0}=0$. Further, $H^{0}(R)_{0}=H_{I}^{0}(A)$ and $H^{0}(A)=A$. Therefore, $H_{I}^{0}(A)=A$ or, equivalently, $I^{n}=0$ for some $n \geq 1$. Thus, if $I \nsubseteq \sqrt{0}$, we must have $a_{1}(R)=-1$. The proof of Theorem 3.1 is now complete.

Corollary 3.2. Let $\ell:=\max \left\{i \mid H_{G_{+}}^{i}(G) \neq 0\right\}$. Then

(i) $a_{\ell}(R)=a_{\ell}(G)$,

(ii) $\ell=\max \left\{i \mid H_{R_{+}}^{i}(R) \neq 0\right\}$ if $I \subseteq \sqrt{0}$ or $\ell \geq 1$.

Proof. For $i \geq \ell$, we have $a_{i}(G) \geq a_{i+1}(G)=-\infty$. Therefore, $a_{i}(R)=a_{i}(G)$ if $i \neq 1$ by Theorem 3.1 (ii). Hence (i) and (ii) are obvious if $\ell>1$. It remains to show that $a_{1}(R)=a_{1}(G)$ if $\ell=1$ or if $\ell=0$ and $I \subseteq \sqrt{0}$. But this follows from Theorem 3.1 (iii).

Example. There are cases where $\max \left\{i \mid H_{G_{+}}^{i}(G) \neq 0\right\} \neq \max \left\{i \mid H_{R_{+}}^{i}(R) \neq 0\right\}$. Let $A=k[x]=k[X] /\left(X-X^{2}\right)$ and $I=(x)$. Then $I^{n}=I$ for all $n \geq 1$. Hence $I \nsubseteq \sqrt{0}$ and $H_{G_{+}}^{i}(G)=0$ for $i>0$, whereas $H_{R_{+}}^{1}(R) \neq 0$ by Theorem 3.1 (iv).

Remark. If $A$ is a local ring, then $\ell=\ell\left(G_{+}\right)$, the analytic spread of $G_{+}$(see the remark following Lemma 2.5). Note that $\ell\left(G_{+}\right)=\ell(I)$, the analytic spread of $I$, which is defined to be the dimension of the graded ring $\bigoplus_{n \geq 0} I^{n} / \mathfrak{m} I^{n}$, where $\mathfrak{m}$ is the maximal ideal of $A$. 
In particular, we can derive from Theorem 3.1 the following result which was proved by Ooishi by a different method [20, Lemma 4.8]. This result was recently rediscovered by Johnson and Ulrich [15, Proposition 4.1].

Corollary 3.3. Let $A$ be a ring and $I$ an ideal of $A$. Then

$$
\operatorname{reg} R_{I}(A)=\operatorname{reg} G_{I}(A) .
$$

Proof. By Theorem 3.1 (i), $a_{i}(R)+i \leq a_{i}(G)+i$ for $i \neq 1$. By Theorem 3.1 (iii) and (iv), either $a_{1}(R)+1 \leq a_{1}(G)+1$ or $a_{1}(R)+1=0 \leq \operatorname{reg} G$. Therefore,

$$
\operatorname{reg} R=\max \left\{a_{i}(R)+i \mid i \geq 0\right\} \leq \max \left\{a_{i}(G)+i \mid i \geq 0\right\}=\operatorname{reg} G .
$$

To prove the converse inequality, choose $i$ maximal such that $a_{i}(G)+i=\operatorname{reg} G$. Then $H_{G_{+}}^{i}(G) \neq 0$ and $a_{i}(G)>a_{i+1}(G)$. Now, using Theorem 3.1 (ii) and (iii), we get $a_{i}(R)=a_{i}(G)$. Hence $\operatorname{reg} G=a_{i}(R)+i \leq \operatorname{reg} R$.

Remark. If $A$ is a local ring, we can apply the above method to study the relationship between the invariants $a_{i}^{*}(R)$ and $a_{i}^{*}(G)$ which characterize the homogeneous vanishing of the local cohomology of $R$ and $G$ with respect to their maximal graded ideals (see the remark following Theorem 2.4). It can be shown that $a_{i}^{*}(R) \leq \max \left\{0, a_{i}^{*}(G)\right\}$ for all $i \geq 0$. In particular, if $A$ is a Cohen-Macaulay ring, then $a_{i}^{*}(R) \leq a_{i}^{*}(G)$, and for $i<\operatorname{dim} A$ we have $a_{i}^{*}(R)=a_{i}^{*}(G)$ if $a_{i}^{*}(G) \geq a_{i+1}^{*}(G)$; cf. [16].

There is also a similar relationship between the Koszul homology of the Rees algebras and the associated graded ring.

Proposition 3.4. Let $A$ be a ring and $I$ an ideal of $A$. Let $J$ be any reduction of I. Let $Q$ denote the ideal generated by $J t$ of $R$ and $J^{*}$ the ideal of $G$ generated by the initial forms of degree 1 of the elements of $J$. Assume that $H_{i}\left(J^{*}, G\right) \neq 0$ for some integer $i \geq 0$. Then

(i) $a\left(H_{i}(Q, R)\right) \leq a\left(H_{i}\left(J^{*}, G\right)\right)$,

(ii) $a\left(H_{i}(Q, R)\right)=a\left(H_{i}\left(J^{*}, G\right)\right)$ if

$$
a\left(H_{i}\left(J^{*}, G\right)\right) \geq a\left(H_{i-1}\left(J^{*}, G\right)\right) \text { and } H_{i-1}\left(J^{*}, G\right) \neq 0 .
$$

Proof. For short we set $H_{j}()=.H_{j}(Q,),. j \geq 0$. Note that $H_{j}\left(J^{*}, G\right)=H_{j}(G)$ and that $H_{j}(A)_{n}=0$ for $n>j$. From (1) we get an isomorphism $H_{j}\left(R_{+}\right)_{n} \simeq$ $H_{j}(R)_{n}, \quad n>j+1$. From (2) we get the exact sequence

$$
H_{i}\left(R_{+}\right)_{n+1} \rightarrow H_{i}(R)_{n} \rightarrow H_{i}(G)_{n} \rightarrow H_{i-1}\left(R_{+}\right)_{n+1} .
$$

Put $m=a\left(H_{i}(G)\right)$. It is well-known that $\left[H_{i}(G)\right]_{n}=0$ for $n<i$. Since $H_{i}(G) \neq 0$, $m \geq i$. For $n>m$, we may replace $H_{i}\left(R_{+}\right)_{n+1}$ by $H_{i}(R)_{n+1}$ and obtain a surjective map $H_{i}(R)_{n+1} \rightarrow H_{i}(R)_{n}$, because $H_{i}(G)_{n}=0$. Since $H_{i}(R)_{n}=0$ for all large $n$, we can successively deduce that $H_{i}(R)_{n}=0$ for $n>m$. Therefore $a\left(H_{i}(R)\right) \leq m$. If $m \geq a\left(H_{i-1}(G)\right)$, then $m \geq a\left(H_{i-1}(R)\right)$. Hence $H_{i-1}\left(R_{+}\right)_{m+1} \simeq H_{i-1}(R)_{m+1}=0$. Therefore, the map $H_{i}(R)_{m} \rightarrow H_{i}(G)_{m}$ is surjective. Since $H_{i}(G)_{m} \neq 0, H_{i}(R)_{m} \neq$ 0 . So we can conclude that $a\left(H_{i}(R)\right)=m$.

As a consequence we obtain the following result of Planas-Vilanova [21, Proposition 5.4]. Note that if $I$ is generated by a regular sequence, then reltype $R_{I}(A)=1$ and reltype $G_{I}(A)=-\infty$. 
Corollary 3.5. Let $A$ be a local ring and $I$ an ideal of $A$ which is not generated by a regular sequence. Then

$$
\text { reltype } R_{I}(A)=\operatorname{reltype} G_{I}(A) .
$$

Proof. By Proposition 2.6,

$$
\text { reltype } R=a\left(H_{1}\left(R_{+}, R\right)\right) \text { and } \quad \operatorname{reltype} G=a\left(H_{1}\left(G_{+}, G\right)\right) \text {. }
$$

By the assumption, $G_{+}$is not generated by a regular sequence. Therefore $H_{1}\left(G_{+}, G\right)$ $\neq 0$. Since $H_{0}\left(G_{+}, G\right)=G / G_{+}, a\left(H_{0}\left(G_{+}, G\right)\right)=0 \leq a\left(H_{1}\left(G_{+}, G\right)\right)$. Thus, using Proposition 3.4 (ii) we get $a\left(H_{1}\left(G_{+}, G\right)\right)=a\left(H_{1}\left(R_{+}, R\right)\right)$.

We conclude this section with the following observation on the behavior of homogeneous filter-regular sequences of $R_{I}(A)$ and $G_{I}(A)$.

Lemma 3.6. Let $x_{1}, \ldots, x_{s}$ be a sequence of elements of $I$. For a given positive integer $d_{i}$, let $y_{i}$ denote the image of $x_{i}$ in $I^{d_{i}} / I^{d_{i}+1}$. Assume that $y_{1}, \ldots, y_{s}$ is a filter-regular sequence of $G_{I}(A)$. Then $x_{1} t^{d_{1}}, \ldots, x_{s} t^{d_{s}}$ is a filter-regular sequence of $R_{I}(A)$.

Proof. By the definition of filter-regular sequences we may assume that $A$ is a local ring. Let $z_{i}=x_{i} t^{d_{i}}, i=1, \ldots, s$. By induction we may assume that $z_{1}, \ldots, z_{s-1}$ is a filter-regular sequence of $R$. If $z_{1}, \ldots, z_{s}$ is not a filter-regular sequence of $R$, there exists an associated prime ideal $P$ of $\left(z_{1}, \ldots, z_{s-1}\right), P \nsupseteq R_{+}$, such that $z_{s} \in P$. Since $P_{n} \neq R_{n}=I^{n}$ for all $n \geq 0,(I, P)_{n}=P_{n}+I^{n+1} \neq I^{n}=R_{n}$ by Nakayama's Lemma. It follows that $\sqrt{(I, P)} \nsupseteq R_{+}$. Hence we can find a prime ideal $Q \supseteq(I, P), Q \nsupseteq R_{+}$. Let $x \in I$ such that $x t \notin Q$. Since $(0: x) x t=0$, $(0: x) R_{Q}=0$. Hence $x$ is a non-zerodivisor in $R_{Q}$. Note that $G=R / I R$ and that $y_{1}, \ldots, y_{s}$ are the images of $z_{1}, \ldots, z_{s}$ in $G$. Then, using the definition of a filter-regular sequence, we can deduce that $z_{1}, \ldots, z_{s}$ form a regular sequence in $R_{Q} / I R_{Q}=R_{Q} / x R_{Q}$. Thus, $x, z_{1}, \ldots, z_{s}$ and therefore $z_{1}, \ldots, z_{s}$ are regular sequences of $R_{Q}$. As a consequence, $z_{1}, \ldots, z_{s}$ is also a regular sequence in $R_{P}$, which contradicts the choice of $P$.

Example. The converse of Lemma 3.6 is not true. Let

$$
A=k[x, u]=k[X, U] /\left(X^{2}-U^{3}\right) \text { and } I=(x, u) A .
$$

Then $G_{I}(A) \simeq k[X, U] /\left(X^{2}\right)$. Hence the image of $x$ in $I / I^{2}$ is not a filter-regular element of $G_{I}(A)$, whereas $x t$ is a non-zerodivisor of $R_{I}(A)$.

\section{Filter-Regular Sequences of Degree 1 \\ OF THE REES ALGEBRA AND THE ASSOCIATED GRADED RING}

In this section we will describe filter-regular sequences of homogeneous elements of degree 1 of $R=R_{I}(A)$ and $G=G_{I}(A)$. The aim is to give characterizations for the Castelnuovo regularity $\operatorname{reg} R_{I}(A)$ and $\operatorname{reg} G_{I}(A)$ in terms of any given reduction of $I$.

Lemma 4.1. Let $x_{1}, \ldots, x_{s}$ be elements of $I$. Then $\mathbf{z}=x_{1} t, \ldots, x_{s} t$ is a filterregular sequence of $R_{I}(A)$ if and only if for all large $n$,

$$
\left[\left(x_{1}, \ldots, x_{i-1}\right) I^{n}: x_{i}\right] \cap I^{n}=\left(x_{1}, \ldots, x_{i-1}\right) I^{n-1}, \quad i=1, \ldots, s .
$$

If that is the case, $a(\mathbf{z})$ is the least number $r$ such that $(\dagger)$ holds for all $n \geq r+1$. 
Proof. It is known that $x_{1} t, \ldots, x_{s} t$ is a filter-regular sequence if and only if for all large $n$,

$$
\left[\left(x_{1} t, \ldots, x_{i-1} t\right): x_{i} t\right]_{n}=\left(x_{1} t, \ldots, x_{i-1} t\right)_{n}, \quad i=1, \ldots, s,
$$

and $a(\mathbf{z})$ is the least integer $r$ such that this condition holds for $n \geq r+1$. Translating this condition in terms of $x_{1}, \ldots, x_{s}$, we immediately obtain the conclusion.

Proposition 4.2. Let $J=\left(x_{1}, \ldots, x_{s}\right)$ be a reduction of $I$ such that $x_{1} t, \ldots, x_{s} t$ is a filter-regular sequence of $R_{I}(A)$. Then $\operatorname{reg} R_{I}(A)$ is the least number $r \geq r_{J}(I)$ such that $(\dagger)$ holds for $n \geq r+1$.

Proof. Let $\mathbf{z}=x_{1} t, \ldots, x_{s} t$ and $Q=(\mathbf{z})$. It is clear that $Q$ is a reduction for $R_{+}$ and $r_{Q}\left(R_{+}\right)=r_{J}(I)$. By Theorem 2.4, $\operatorname{reg} R=\max \left\{a(\mathbf{z}), r_{J}(I)\right\}$. Therefore, the conclusion follows from Lemma 4.1.

Remark. The case $\left(x_{1}, \ldots, x_{s}\right)=I$ of $(\dagger)$ was already considered by Costa [2] (for $r=0$ ) and Raghavan [22] (for arbitrary $r$ ) without knowing the relevance of this condition to filter-regular sequences. They proved that if $(\dagger)$ holds for all $n \geq r+1$, then $I$ is an ideal of type $\leq r+1$, i.e. reltype $R_{I}(A) \leq r+1$. But this is obvious because reltype $R_{I}(A) \leq \operatorname{reg} R_{I}(A)+1$ and $\operatorname{reg} R_{I}(A) \leq r$ by Proposition 4.2.

In the following we will denote by $x^{*}$ the image of an element $x \in I$ in $I / I^{2}$. Similarly as for Lemma 4.1, we can prove the following characterization of a homogeneous filter-regular sequence of degree 1 of $G_{I}(A)$.

Lemma 4.3. Let $x_{1}, \ldots, x_{s}$ be elements of $I$. Then $\mathbf{x}^{*}=x_{1}^{*}, \ldots, x_{s}^{*}$ is a filterregular sequence of $G_{I}(A)$ if and only if for all large $n$,

$$
\begin{aligned}
& \left\{\left[\left(x_{1}, \ldots, x_{i-1}\right) I^{n}+I^{n+2}\right]: x_{i}\right\} \cap I^{n} \\
& \quad=\left(x_{1}, \ldots, x_{i-1}\right) I^{n-1}+I^{n+1}, \quad i=1, \ldots, s .
\end{aligned}
$$

If that is the case, $a\left(\mathbf{x}^{*}\right)$ is the least number $r$ such that $(\ddagger)$ holds for $n \geq r+1$.

One can derive from Lemma 4.3 a characterization for $\operatorname{reg} G_{I}(A)$ similar to that of Proposition 4.2. But we will see that there is a simpler condition which does not depend on the asymptotic behavior of $I$. We shall need the following observations.

Lemma 4.4. Let $r=\operatorname{reg} G_{I}(A)$. Then the following conditions hold for any element $x$ of $I$ for which $x^{*}$ is a filter-regular element of $G_{I}(A)$ :

(i) $(x) \cap I^{n}=x I^{n-1}$ for $n \geq r+1$.

(ii) $\operatorname{reg} G_{I /(x)}(A /(x)) \leq r$.

Proof. Let $\bar{G}=G_{I /(x)}(A /(x))$. For all $n \geq 0$ we have

$$
\bar{G}_{n}=I^{n}+(x) / I^{n+1}+(x)=I^{n} / I^{n+1}+(x) \cap I^{n} .
$$

Since $\left[G /\left(x^{*}\right)\right]_{n}=I^{n} / I^{n+1}+x I^{n-1}$, there is an exact sequence of graded $G$-modules

$$
0 \rightarrow K \rightarrow G /\left(x^{*}\right) \rightarrow G_{I /(x)}(A /(x)) \rightarrow 0
$$

with $K_{n}=I^{n+1}+(x) \cap I^{n} / I^{n+1}+x I^{n-1}$. By the Artin-Rees lemma, there is an integer $c$ such that for all $n \geq c,(x) \cap I^{n} \subseteq x I^{n-c}$; hence $I^{n}: x \subseteq(0: x)+I^{n-c}$. By Lemma 4.3, $\left(I^{m}: x\right) \cap I^{m-2}=I^{m-1}$ for all large $m$. Applying this formula for $m=n-c+2, \ldots, n$ we obtain, for all large $n$,

$$
\left(I^{n}: x\right) \cap I^{n-c}=\left(I^{n}: x\right) \cap I^{n-c+1}=\ldots=\left(I^{n}: x\right) \cap I^{n-1}=I^{n-1} .
$$


It follows that $I^{n}: x=(0: x)+I^{n-1}$. Hence $(x) \cap I^{n}=x I^{n-1}$. This implies $K_{n}=0$ for all large $n$.

As a consequence, $K \simeq H_{G_{+}}^{0}\left(G /\left(x^{*}\right)\right)$. By Lemma 2.1,

$$
a_{0}\left(G /\left(x^{*}\right)\right) \leq \max \left\{a_{0}(G), a_{1}(G)+1\right\} \leq r .
$$

Therefore, we must have $K_{n}=0$ for $n \geq r+1$. From this it follows that $(x) \cap I^{n} \subseteq$ $x I^{n-1}+I^{n+1}$. Hence $(x) \cap I^{n}=x I^{n-1}+(x) \cap I^{n+1}=x I^{n-1}+(x) \cap I^{n+2}=\ldots$. But we have seen above that $(x) \cap I^{m}=x I^{m-1}$ for all large $m$. Therefore $(x) \cap I^{n}=$ $x I^{n-1}$ for $n \geq r+1$.

Since $H_{G_{+}}^{i}(K)=0$ for $i \geq 1$, from the above exact sequence we can deduce that there is a surjective map $H_{G_{+}}^{0}\left(G /\left(x^{*}\right)\right) \rightarrow H_{G_{+}}^{0}(\bar{G})$, and $H_{G_{+}}^{i}(\bar{G}) \simeq H_{G_{+}}^{i}\left(G /\left(x^{*}\right)\right)$, $i \geq 1$. By Lemma 2.1, $H_{G_{+}}^{i}\left(G /\left(x^{*}\right)\right)_{n}=0$ for $n \geq r_{i}+1, i \geq 0$. Hence $H_{G_{+}}^{i}(\bar{G})_{n}=0$ for $n \geq r-i+1, i \geq 0$, too. This shows that $\operatorname{reg} \bar{G} \leq r$.

Lemma 4.5. Let $x_{1}, \ldots, x_{s}$ be elements of $I$. Then $x_{1}^{*}, \ldots, x_{s}^{*}$ is a filter-regular sequence of $G_{I}(A)$ if and only if the following conditions are satisfied:

(i) $x_{1}^{*}$ is a filter-regular element of $G_{I}(A)$.

(ii) $x_{2}^{*}, \ldots, x_{s}^{*}$ is a filter-regular sequence of $G_{I /\left(x_{1}\right)}\left(A /\left(x_{1}\right)\right)$.

Proof. We may assume that (i) is satisfied and $s \geq 2$. Then $x_{1}^{*}, \ldots, x_{s}^{*}$ is a filterregular sequence if and only if for all large $n$,

$$
\left[\left(x_{1}^{*}, \ldots, x_{i-1}^{*}\right): x_{i}^{*}\right]_{n}=\left(x_{1}^{*}, \ldots, x_{i-1}^{*}\right)_{n}, i=2, \ldots, s .
$$

Put $\bar{G}=G_{I /\left(x_{1}\right)}\left(A /\left(x_{1}\right)\right)$. By the proof of Lemma $4.4, \bar{G}$ can be considered as a quotient ring of $G /\left(x_{1}^{*}\right)$, and $\bar{G}_{n}=\left[G /\left(x_{1}^{*}\right)\right]_{n}$ for $n \geq \operatorname{reg} G+1$. Hence the above condition is equivalent to the condition that for all large $n$,

$$
\left[\left(x_{2}^{*}, \ldots, x_{i-1}^{*}\right) \bar{G}: x_{i}^{*}\right]_{n}=\left[\left(x_{2}^{*}, \ldots, x_{i-1}^{*}\right) \bar{G}\right]_{n}, \quad i=2, \ldots, s,
$$

which means that $x_{2}^{*}, \ldots, x_{s}^{*}$ form a filter-regular sequence of $\bar{G}$.

We will use the above lemmas to show that there is a simple characterization for $x_{1}^{*}, \ldots, x_{s}^{*}$ to be a filter-regular sequence of $G_{I}(A)$ if $\left(x_{1}, \ldots, x_{s}\right)$ is a reduction of $I$. This characterization is inspired by the following remark.

Remark. Let $S$ be a standard graded ring. Then $G_{S_{+}}(S) \simeq S$. By [24, Lemma 2.1], a sequence $z_{1}, \ldots, z_{s}$ of homogeneous elements of degree 1 of $S$ is filter-regular if and only if for large $n,\left[\left(z_{1}, \ldots, z_{i-1}\right): z_{i}\right]_{n}=\left(z_{1}, \ldots, z_{i}\right)_{n}, i=1, \ldots, s$. This condition can be rewritten as follows: there exists an integer $r$ such that

$$
\left[\left(z_{1}, \ldots, z_{i-1}\right): z_{i}\right] \cap\left(S_{+}\right)^{r+1}=\left(z_{1}, \ldots, z_{i-1}\right)\left(S_{+}\right)^{r}, i=1, \ldots, s .
$$

This remark leads us to consider the similar condition

$$
\left[\left(x_{1}, \ldots, x_{i-1}\right): x_{i}\right] \cap I^{r+1}=\left(x_{1}, \ldots, x_{i-1}\right) I^{r}, \quad i=1, \ldots, s .
$$

Proposition 4.6. Let $x_{1}, \ldots, x_{s}$ be a sequence of elements of $I$ with $x_{1}^{*}, \ldots, x_{s}^{*}$ a filter-regular sequence of $G_{I}(A)$. Then $(\star)$ holds for all integers $r \geq \operatorname{reg} G_{I}(A)$.

Proof. By Lemma 3.6, $x_{1} t$ is a filter-regular element of $R$. Hence, by Lemma 4.1, $\left(0: x_{1}\right) \cap I^{r+1}=0$ for $r \geq a\left(x_{1} t\right)$. By Proposition 2.2 (i) and Corollary 3.3, $a\left(x_{1} t\right) \leq \operatorname{reg} R=\operatorname{reg} G$. Therefore, the case $s=1$ is obvious. For $s \geq 2$ we note that $\operatorname{reg} G \geq \operatorname{reg} G_{I /\left(x_{1}\right)}\left(A /\left(x_{1}\right)\right)$ by Lemma 4.4 (ii). By Lemma $4.5, x_{2}^{*}, \ldots, x_{s}^{*}$ is 
a filter-regular sequence in $G_{I /\left(x_{1}\right)}\left(A /\left(x_{1}\right)\right)$. Using induction, we may assume that for $r \geq \operatorname{reg} G$,

$$
\left[\left(x_{1}, \ldots, x_{i-1}\right): x_{i}\right] \cap\left[\left(x_{1}\right)+I^{r+1}\right]=\left(x_{1}\right)+\left(x_{2}, \ldots, x_{i-1}\right) I^{r}, \quad i=2, \ldots, s .
$$

From this it follows that

$$
\left[\left(x_{1}, \ldots, x_{i-1}\right): x_{i}\right] \cap I^{r+1}=\left(x_{1}\right) \cap I^{r+1}+\left(x_{2}, \ldots, x_{i-1}\right) I^{r}, \quad i=2, \ldots, s .
$$

Since $\left(x_{1}\right) \cap I^{r+1}=x_{1} I^{r}$ by Lemma 4.4 (i), we obtain $(\star)$.

Remark. Using Lemma 4.3 for $s=1$ and then induction, we can show similarly as above that if $x_{1}^{*}, \ldots, x_{s}^{*}$ is a filter-regular sequence, then for $n \geq \operatorname{reg} G_{I}(A)+1$,

$$
\left[\left(x_{1}, \ldots, x_{i-1}, I^{n+2}\right): x_{i}\right] \cap I^{n}=\left(x_{1}, \ldots, x_{i-1}\right) I^{n-1}+I^{n+1}, \quad i=1, \ldots, s .
$$

By Proposition $4.6,(\star)$ is a necessary condition for $x_{1}^{*}, \ldots, x_{s}^{*}$ to be a filterregular sequence of $G_{I}(A)$. If $\left(x_{1}, \ldots, x_{s}\right)$ is a reduction of $I$, we can show that $(\star)$ is also a sufficient condition and that it is closely related to $\operatorname{reg} R_{I}(A)$ and $\operatorname{reg} G_{I}(A)$.

Proposition 4.7. Let $J=\left(x_{1}, \ldots, x_{s}\right)$ be a reduction of $I$ such that the sequence $x_{1}, \ldots, x_{s}$ satisfies the condition $(\star)$ for a fixed integer $r \geq r_{J}(I)$. Then

(i) For all $n \geq r+1$,

$$
\left[\left(x_{1}, \ldots, x_{i-1}\right): x_{i}\right] \cap I^{n}=\left(x_{1}, \ldots, x_{i-1}\right) I^{n-1}, \quad i=1, \ldots, s .
$$

(ii) $x_{1} t, \ldots, x_{s} t$ is a filter-regular sequence of $R_{I}(A)$.

(iii) $x_{1}^{*}, \ldots, x_{s}^{*}$ is a filter-regular sequence of $G_{I}(A)$.

(iv) $\operatorname{reg} R_{I}(A)=\operatorname{reg} G_{I}(A) \leq r$.

Proof . (i) By the assumption we may let $n>r+1$. Then $I^{n}=J I^{n-1}$. Hence

$\left[\left(x_{1}, \ldots, x_{s-1}\right): x_{s}\right] \cap I^{n}=\left(x_{1}, \ldots, x_{s-1}\right) I^{n-1}+x_{s}\left\{\left[\left(x_{1}, \ldots, x_{s-1}\right): x_{s}^{2}\right] \cap I^{n-1}\right\}$.

By induction we may assume that $\left[\left(x_{1}, \ldots, x_{s-1}\right): x_{s}\right] \cap I^{n-1}=\left(x_{1}, \ldots, x_{s-1}\right) I^{n-2}$. From this it follows that $\left[\left(x_{1}, \ldots, x_{s-1}\right): x_{s}^{2}\right] \cap\left(I^{n-1}: x_{s}\right) \subseteq\left(x_{1}, \ldots, x_{s-1}\right): x_{s}$. Hence

$$
\begin{aligned}
{\left[\left(x_{1}, \ldots, x_{s-1}\right): x_{s}^{2}\right] \cap I^{n-1} } & \subseteq\left[\left(x_{1}, \ldots, x_{s-1}\right): x_{s}\right] \cap I^{n-1} \\
& =\left(x_{1}, \ldots, x_{s-1}\right) I^{n-2} .
\end{aligned}
$$

This implies $x_{s}\left\{\left[\left(x_{1}, \ldots, x_{s-1}\right): x_{s}^{2}\right] \cap I^{n-1}\right\} \subseteq\left(x_{1}, \ldots, x_{s}\right) I^{n-1}$. Therefore,

$$
\left[\left(x_{1}, \ldots, x_{s-1}\right): x_{s}\right] \cap I^{n}=\left(x_{1}, \ldots, x_{s-1}\right) I^{n-1} .
$$

For $i<s-1$, using induction on $n$ and on $i$ we have

$$
\begin{aligned}
{\left[\left(x_{1}, \ldots, x_{i-1}\right): x_{i}\right] \cap I^{n} } & \subset\left\{\left[\left(x_{1}, \ldots, x_{i-1}\right): x_{i}\right] \cap I^{n-1}\right\} \cap I^{n} \\
& =\left(x_{1}, \ldots, x_{i-1}\right) I^{n-2} \cap I^{n} \subset\left(x_{1}, \ldots, x_{i}\right) \cap I^{n} \\
& \subset\left[\left(x_{1}, \ldots, x_{i}\right): x_{i+1}\right] \cap I^{n}=\left(x_{1}, \ldots, x_{i}\right) I^{n-1} .
\end{aligned}
$$

It follows that

$$
\left[\left(x_{1}, \ldots, x_{i-1}\right): x_{i}\right] \cap I^{n}=\left(x_{1}, \ldots, x_{i-1}\right) I^{n-1}+x_{i}\left\{\left[\left(x_{1}, \ldots, x_{i-1}\right): x_{i}^{2}\right] \cap I^{n-1}\right\} .
$$

Similarly as for the case $i=s$, we can then show that

$$
\left[\left(x_{1}, \ldots, x_{i-1}\right): x_{i}\right] \cap I^{n}=\left(x_{1}, \ldots, x_{i-1}\right) I^{n-1} .
$$

(ii) From (i) we see that condition ( $\dagger$ ) of Lemma 4.1 is satisfied. Therefore, the conclusion follows from Lemma 4.1. 
(iii) If $s=1, I^{n+2}=x_{1} I^{n+1}$ for $n \geq r$. Hence $I^{n+2}: x_{1}=I^{n+1}+\left(0: x_{1}\right)$. Using (i) we get

$$
\left(I^{n+2}: x_{1}\right) \cap I^{n}=I^{n+1}+\left(0: x_{1}\right) \cap I^{n}=I^{n+1} .
$$

By Lemma 4.3, this implies that $x_{1}^{*}$ is a filter-regular element of $G$. If $s \geq 2$, using (i) we have $\left(x_{1}\right) \cap I^{n+2}=x_{1} I^{n+1}$ for $n \geq r-1$. From this it follows that $I^{n+2}: x_{1}=\left(0: x_{1}\right)+I^{n+1}$. As above, we can then deduce that $x_{1}^{*}$ is a filter-regular element of $G$. Further, from $(\star)$ it follows that

$$
\left[\left(x_{1}, x_{2}, \ldots, x_{i-1}\right): x_{i}\right] \cap\left(x_{1}, I^{n}\right)=\left(x_{1}\right)+\left(x_{2}, \ldots, x_{i-1}\right) I^{n-1}, \quad i=2, \ldots, s .
$$

By induction we may assume that the images of $x_{2}^{*}, \ldots, x_{s}^{*}$ form a filter-regular sequence in $G_{I /\left(x_{1}\right)}\left(A /\left(x_{1}\right)\right)$. Hence $x_{1}^{*}, \ldots, x_{s}^{*}$ is a filter-regular sequence of $G$ by Lemma 4.5 .

(iv) From (i) we see that $(\dagger)$ is satisfied. Hence $\operatorname{reg} R \leq r$ by Proposition 4.2. The equality $\operatorname{reg} R=\operatorname{reg} G$ follows from Corollary 3.3.

The proof of Proposition 4.7 is now complete.

The above results give characterizations for $\operatorname{reg} G_{I}(A)$ and $\operatorname{reg} R_{I}(A)$ which, unlike Proposition 4.2, do not depend on the asymptotic behavior of $I$.

Theorem 4.8. Let $J=\left(x_{1}, \ldots, x_{s}\right)$ be a reduction for $I$. Then $x_{1}^{*}, \ldots, x_{s}^{*}$ is a filter-regular sequence of $G_{I}(A)$ if and only if the following condition holds for some fixed integer $r \geq r_{J}(I)$ :

$$
\left[\left(x_{1}, \ldots, x_{i-1}\right): x_{i}\right] \cap I^{r+1}=\left(x_{1}, \ldots, x_{i-1}\right) I^{r}, \quad i=1, \ldots, s .
$$

If that is the case, $\operatorname{reg} R_{I}(A)$ resp. $\operatorname{reg} G_{I}(A)$ is the least integer $r$ with this property.

Proof. The assertions follow from Proposition 4.6 and Proposition 4.7 (iii) and (iv).

Remark. The condition $(\star)$ can be replaced by the condition

$$
\left[\left(x_{1}, \ldots, x_{i-1}\right) I^{r}: x_{i}\right] \cap I^{r+1}=\left(x_{1}, \ldots, x_{i-1}\right) I^{r}, \quad i=1, \ldots, s .
$$

That $(\star)$ implies $(*)$ is obvious. Conversely, dividing $(*)$ by $x_{i}^{n}$, we get

$$
\left[\left(x_{1}, \ldots, x_{i-1}\right) I^{r}: x_{i}^{n+1}\right] \cap I^{r+1}=\left[\left(x_{1}, \ldots, x_{i-1}\right) I^{r}: x_{i}^{n}\right] \cap I^{r+1}
$$

for $n=1, \ldots, r$. From this it follows that

$$
\left[\left(x_{1}, \ldots, x_{i-1}\right) I^{r}: x_{i}^{r+1}\right] \cap I^{r+1}=\left[\left(x_{1}, \ldots, x_{i-1}\right) I^{r}: x_{i}\right] \cap I^{r+1} .
$$

Since $\left(x_{1}, \ldots, x_{i-1}\right) I^{r}: x_{i} \subseteq\left(x_{1}, \ldots, x_{i-1}\right): x_{i} \subseteq\left(x_{1}, \ldots, x_{i-1}\right) I^{r}: x_{i}^{r+1}$, we see that $(*)$ implies $(\star)$. The condition $(\star)$ has been considered in [2] and [22] in the case $I=\left(x_{1}, \ldots, x_{s}\right)$.

Theorem 4.9. Let $A$ be a local ring with infinite residue field and $I$ an ideal of $A$. Let $J$ be any reduction for I generated by forms of degree 1. For a fixed integer $r \geq 0$, the following conditions are equivalent:

(i) $\operatorname{reg} R_{I}(A)=r$.

(ii) $\operatorname{reg} G_{I}(A)=r$.

(iii) There exists a minimal basis $x_{1}, \ldots, x_{s}$ for $J$ such that $(\star)$ holds and $r$ is the least number $\geq r_{J}(I)$ with this property.

Proof. (i) $\Leftrightarrow$ (ii) is a consequence of Corollary 3.3. (ii) $\Leftrightarrow$ (iii) follows from Theorem 4.8 , because by Lemma 2.5 we can find a minimal basis $x_{1}, \ldots, x_{s}$ for $J$ such that $x_{1}^{*}, \ldots, x_{s}^{*}$ is a filter-regular sequence of $G$. 


\section{Sequences of Regular tyPe}

For convenience we call a sequence $x_{1}, \ldots, x_{s}$ of elements of $A$ a $d$-sequence if

$$
\left[\left(x_{1}, \ldots, x_{i-1}\right): x_{i}\right] \cap I=\left(x_{1}, \ldots, x_{i-1}\right), \quad i=1, \ldots, s,
$$

where $I=\left(x_{1}, \ldots, x_{s}\right)$, without requiring that $x_{1}, \ldots, x_{s}$ is a minimal basis for $I$. This notion can be naturally generalized as follows.

Let $r$ be any non-negative integer. A sequence $x_{1}, \ldots, x_{s}$ of elements of a commutative noetherian ring $A$ is called a sequence of regular type $r$ if for $I=\left(x_{1}, \ldots, x_{s}\right)$,

$$
\left[\left(x_{1}, \ldots, x_{i}\right): x_{i+1}\right] \cap I^{r+1}=\left(x_{1}, \ldots, x_{i}\right) I^{r}, \quad i=0, \ldots, s-1,
$$

and $r$ is the smallest integer with this property (cf. [6], [13], and [22] for other generalizations of $d$-sequences). The name stems from the following result.

Theorem 5.1. Let $I$ be an arbitrary ideal of a ring A. Then:

(i) $\operatorname{reg} R_{I}(A)=\operatorname{reg} G_{I}(A)=r$ if $I$ is generated by a sequence of regular type $r$.

(ii) $I$ is generated by a sequence of regular type $r$ if $A$ is a local ring with infinite residue field and $\operatorname{reg} R_{I}(A)=r$ or $\operatorname{reg} G_{I}(A)=r$.

Proof. The assertions follow from Theorem 4.8 and Theorem 4.9, respectively.

Remark. By Proposition 4.7 (i), if $x_{1}, \ldots, x_{s}$ is a sequence of regular type $r$, then for all $n \geq r+1$,

$$
\left[\left(x_{1}, \ldots, x_{i-1}\right): x_{i}\right] \cap I^{n}=\left(x_{1}, \ldots, x_{i-1}\right) I^{n-1}, \quad i=1, \ldots, s .
$$

By Theorem $4.8, x_{1}, \ldots, x_{s}$ is a sequence of regular type $r$ if and only if $x_{1}^{*}, \ldots, x_{s}^{*}$ is a filter-regular sequence of $G_{I}(A)$ and $\operatorname{reg} G_{I}(A)=r$. Hence sequences of regular types enjoy many interesting properties which can be derived from those of filterregular sequences of $G_{I}(A)$ (see Section 4 ).

The most interesting property of an ideal $I$ generated by a $d$-sequence is that it is of linear type, i.e. reltype $R_{I}(A)=1$ [11], [28]. By Proposition 2.6, $\operatorname{reg} R_{I}(A)$ is the maximum of certain invariants of $R_{I}(A)$ which include reltype $R_{I}(A)-1$. Hence the following consequence of Theorem 5.1 clearly distinguishes ideals generated by $d$-sequences from ideals of linear types.

Corollary 5.2. Let $I$ be an arbitrary ideal of a ring A. Then:

(i) $\operatorname{reg} R_{I}(A)=\operatorname{reg} G_{I}(A)=0$ if $I$ is generated by a d-sequence.

(ii) $I$ is generated by a $d$-sequence if $A$ is a local ring with infinite residue field and $\operatorname{reg} R_{I}(A)=0$ or $\operatorname{reg} G_{I}(A)=0$.

The statements concerning $\operatorname{reg} G_{I}(A)=0$ are implicitly contained in [7, Lemma 12.7 and Theorem 12.10] and [17, (2.3) and (2.20)].

We shall see that Theorem 5.1 has a graded version.

Lemma 5.3. Let $S$ be a standard graded ring. Let $\mathbf{z}=z_{1}, \ldots, z_{s}$ be a sequence of homogeneous elements of degree 1 such that $S_{+}=\left(z_{1}, \ldots, z_{s}\right)$. Then $\mathbf{z}$ is a sequence of regular type $r$ if and only if $\mathbf{z}$ is a filter-regular sequence and $\operatorname{reg} S=r$.

Proof. Let $r=\operatorname{reg} S$. By Theorem 2.4, $\mathbf{z}$ is a filter-regular sequence if and only if $a(\mathbf{z})=r$, i.e. $\left[\left(z_{1}, \ldots, z_{i-1}\right): z_{i}\right]_{n}=\left(z_{1}, \ldots, z_{i-1}\right)_{n}, i=1, \ldots, s$, for $n \geq r+1$ and $r$ is the smallest integer with this property. Since $z_{1}, \ldots, z_{s}$ are homogeneous elements of degree 1 , this condition is equivalent to the condition

$$
\left[\left(z_{1}, \ldots, z_{i-1}\right): z_{i}\right] \cap\left(S_{+}\right)^{r+1}=\left(z_{1}, \ldots, z_{i-1}\right)\left(S_{+}\right)^{r}, \quad i=1, \ldots, s .
$$


Theorem 5.4. Let $S=\bigoplus_{n \geq 0} S_{n}$ be a standard graded ring. Then:

(i) $\operatorname{reg} S=r$ if $S_{+}$is generated by a sequence of regular type $r$.

(ii) $S_{+}$is generated by a sequence of regular type $r$ if $S_{0}$ is a local ring with infinite residue field and $\operatorname{reg} S=r$.

Proof. (i) Since $S$ is standard graded, $G_{S_{+}}(S) \simeq S$. Hence the assertion is a consequence of Theorem 5.1 (i).

(ii) By Lemma 2.5, there is a filter-regular sequence of homogeneous elements of degree 1 which minimally generate $S_{+}$. Hence the statement follows from Lemma 5.3 .

Recall that a standard graded ring $S$ is called special graded if the ideal $S_{+}$is generated by a $d$-sequence [12]. From Theorem 5.4 we obtain the following characterization of special graded rings in terms of the Castelnuovo regularity. This characterization can be also deduced from results of Herzog, Simis, and Vasconcelos [7, Lemma 12.7 and Lemma 12.8] or Kühl [17, (2.3)] by using the characterization of the Castelnuovo regularity in terms of the vanishing of Koszul homology (Proposition 2.6).

Corollary 5.5. Let $S=\bigoplus_{n \geq 0} S_{n}$ be a standard graded ring. Then $\operatorname{reg} S=0$ if $S$ is special graded. The converse is true if $S_{0}$ is a local ring with infinite residue field.

The following interplay between sequences of regular types of $A, R_{I}(A)$, and $G_{I}(A)$ is inspired by Huneke's results on special graded algebras [12].

Proposition 5.6. Let $x_{1}, \ldots, x_{s}$ be elements of a ring $A$ and $I=\left(x_{1}, \ldots, x_{s}\right)$. For a fixed integer $r \geq 0$, the following conditions are equivalent:

(i) $x_{1}, \ldots, x_{s}$ is a sequence of regular type $r$.

(ii) $x_{1} t, \ldots, x_{s} t$ is a sequence of regular type $r$ in $R_{I}(A)$ and $x_{1}^{*}, \ldots, x_{s}^{*}$ is a filter-regular sequence of $G_{I}(A)$.

(iii) $x_{1}^{*}, \ldots, x_{s}^{*}$ is a sequence of regular type $r$ in $G_{I}(A)$.

Proof. (i) $\Rightarrow$ (ii): By Theorem 4.6, $x_{1} t, \ldots, x_{s} t$ and $x_{1}^{*}, \ldots, x_{s}^{*}$ are filter-regular sequence of $R$ and $G$, respectively, and $\operatorname{reg} R=r$. Thus, by Lemma $5.3, x_{1} t, \ldots, x_{s} t$ is a sequence of regular type $r$ in $R$.

(ii) $\Rightarrow$ (iii): By Lemma 5.3, $\operatorname{reg} R=r$. Hence $\operatorname{reg} G=r$ by Corollary 3.3. Applying Lemma 5.3 once more, we see that $x_{1}^{*}, \ldots, x_{s}^{*}$ is a sequence of regular type $r$.

(iii) $\Rightarrow$ (i): By Lemma 5.3, $x_{1}^{*}, \ldots, x_{s}^{*}$ is a filter-regular sequence of $G$ and $\operatorname{reg} G=r$. By Theorem 4.8, this implies that $x_{1}, \ldots, x_{s}$ is a sequence of regular type $r$.

Corollary 5.7. Let $x_{1}, \ldots, x_{s}$ be elements of $a$ ring $A$ and $I=\left(x_{1}, \ldots, x_{s}\right)$. Then the following conditions are equivalent:

(i) $x_{1}, \ldots, x_{s}$ is a d-sequence.

(ii) $x_{1} t, \ldots, x_{s} t$ is a $d$-sequence of $R_{I}(A)$ and $x_{1}^{*}, \ldots, x_{s}^{*}$ is a filter-regular sequence of $G_{I}(A)$.

(iii) $x_{1}^{*}, \ldots, x_{s}^{*}$ is a d-sequence of $G_{I}(A)$.

Remark. The implication (i) $\Rightarrow$ (ii) and (iii) has been proved by Huneke $[12$, Corollary 1.1 and Theorem 1.2]. The equivalence (i) $\Leftrightarrow$ (iii) was shown by Kühl [17, $(2.20)]$ (see also [17, Theorem 2.10]). But the equivalence (i) $\Leftrightarrow$ (ii) is new. 


\section{Some APPLiCATiOns}

The first application concerns a recent result of Marley [18, Theorem 2.1] comparing certain invariants $a_{i}\left(G_{I}(A)\right)$ for an $\mathfrak{m}$-primary ideal $I$ of a Cohen-Macaulay local ring $(A, \mathfrak{m})$. We shall see that his result is a natural consequence of the relationship between $a_{i}\left(R_{I}(A)\right)$ and $a_{i}\left(G_{I}(A)\right)$. The following generalized version of Marley's result is due to Hoa [8, Theorem 5.2] (see also [5, Lemma 4.5] for a similar result).

Proposition 6.1. Let $A$ be a local ring and $I$ an ideal of $A$. Set $G=G_{I}(A)$ and $s=$ grade $G_{+}$. Assume that $s \leq \operatorname{grade} I-1$. Then $a_{s}(G)<a_{s+1}(G)$.

Proof. Without restriction we may assume that the residue field of $A$ is infinite. Then there is a regular sequence $x_{1}, \ldots, x_{s}$ of elements in $I \backslash I^{2}$ such that $x_{1}^{*}, \ldots, x_{s}^{*}$ is a regular sequence of $G$. Put $\bar{A}=A /\left(x_{1}, \ldots, x_{s}\right)$ and $\bar{I}=I /\left(x_{1}, \ldots, x_{s}\right)$. Let $\bar{G}=G_{\bar{I}}(\bar{A})$ and $\bar{R}=R_{\bar{I}}(\bar{A})$. Then $\bar{G}=G /\left(x_{1}^{*}, \ldots, x_{s}^{*}\right)$. By Corollary 2.4, $a_{i}(G)=0, i=0, \ldots, s-1$, and $a_{s}(G) \geq-s$. Using Proposition 2.2 (ii), we can deduce that

$$
\begin{aligned}
a_{s}(G)+s & =a_{0}(\bar{G}) \geq 0, \\
\max \left\{a_{s}(G)+s, a_{s+1}(G)+s+1\right\} & =\max \left\{a_{0}(\bar{G}), a_{1}(\bar{G})+1\right\} .
\end{aligned}
$$

If $a_{s}(G) \geq a_{s+1}(G)$, then $a_{0}(\bar{G}) \geq a_{1}(\bar{G})$. By Theorem 3.1 (ii), $a_{0}(\bar{R})=a_{0}(\bar{G}) \geq 0$. Since grade $\bar{I}=\operatorname{grade} I-s>0$, we have $H_{\bar{I}}^{0}(\bar{A})=0$. From this it follows that $H_{\bar{R}_{+}}^{0}(\bar{R})=0$. So we obtain $a_{0}(\bar{R})=-\infty$, a contradiction.

Remark. Marley's result consists of two parts. The first part has been modified as above. The second part can be generalized as follows. Assume that $s \leq$ grade $I-2$. Then either $a_{s+1}(G)<a_{s+2}(G)$ or

$$
a_{s+1}(G)=\max \left\{n \mid\left(x_{1}, \ldots, x_{s}, I^{n+s+1}\right): x_{s+1} \neq\left(x_{1}, \ldots, x_{s}, I^{n+s}\right)\right\},
$$

where $x_{1}, \ldots, x_{s+1}$ is a superficial sequence of $I$. The proof is more complicated but follows the same line as the above proof for Proposition 6.1; hence we omit it.

Recall that an element $x \in I \backslash I^{2}$ is a superficial element (of order 1) for $I$ if there is an integer $c$ such that $\left(I^{n}: x\right) \cap I^{c}=I^{n-1}$ for all large $n$ [29]. This notion has played an important role in the theory of multiplicity. A sequence $x_{1}, \ldots, x_{s}$ of elements of $I$ is a superficial sequence of $I$ if $x_{i}$ is a superficial element of $A /\left(x_{1}, \ldots, x_{i-1}\right), i=1, \ldots, s$. The obstacle to finding superficial sequences is that one needs to check certain relations for all large $n$. We can circumvent this by using the notion of a filter-regular element.

Lemma 6.2. Let $x_{1}, \ldots, x_{s}$ be elements of $I$. Then $x_{1}, \ldots, x_{s}$ is a superficial sequence of $I$ if and only if the following conditions are satisfied:

(i) $x_{i} \notin\left(x_{1}, \ldots, x_{i-1}\right)+I^{2}, i=1, \ldots, s$.

(ii) $x_{1}^{*}, \ldots, x_{s}^{*}$ is a filter-regular sequence of $G_{I}(A)$.

Proof. We may assume that (i) is satisfied. If $x_{1}$ is a superficial element of $I$, then there is an integer $c$ such that $\left(I^{n+2}: x_{1}\right) \cap I^{c}=I^{n+1}$ for all large $n$. From this it follows that $\left(I^{n+2}: x_{1}\right) \cap I^{n}=I^{n+1}$. Hence $x_{1}^{*}$ is a filter-regular element of $G_{I}(A)$ by Lemma 4.3. Conversely, if there is an integer $c$ such that $\left(I^{n+2}: x_{1}\right) \cap I^{n}=I^{n+1}$ for all $n \geq c$, we can deduce that for $n \geq c+2$,

$$
\left(I^{n}: x_{1}\right) \cap I^{c}=\left(I^{n}: x_{1}\right) \cap I^{c+1}=\ldots=\left(I^{n}: x_{1}\right) \cap I^{n-2}=I^{n-1} .
$$


This proves the case $s=1$. If $s \geq 2$, we may further assume that $x_{1}$ is a superficial element of $I$ or, equivalently, $x_{1}^{*}$ is a filter-regular sequence of $G_{I}(A)$. By Lemma $4.5, x_{1}^{*}, \ldots, x_{s}^{*}$ is a filter-regular sequence if and only if $x_{2}^{*}, \ldots, x_{s}^{*}$ is a filter-regular sequence of $G_{I /\left(x_{1}\right)}\left(A /\left(x_{1}\right)\right)$. By induction, this condition holds if and only if $x_{2}, \ldots, x_{s}$ is a superficial sequence of the ideal $I /\left(x_{1}\right)$.

For a superficial sequence which generates a reduction of $I$ we have the following characterization which does not depend on the asymptotic behavior of the elements. Note that if $A$ is a local ring with infinite residue field, any superficial element of $I$ can be extended to such a superficial sequence.

Proposition 6.3. Let $x_{1}, \ldots, x_{s}$ be elements of I which generate a reduction $J$ of $I$. Then $x_{1}, \ldots, x_{s}$ is a superficial sequence of $I$ if and only if the following conditions are satisfied:

(i) $x_{i} \notin\left(x_{1}, \ldots, x_{i-1}\right)+I^{2}, i=1, \ldots, s$.

(ii) There is an integer $r \geq r_{J}(I)$ such that

$$
\left[\left(x_{1}, \ldots, x_{i-1}\right): x_{i}\right] \cap I^{r+1}=\left(x_{1}, \ldots, x_{i-1}\right) I^{r}, \quad i=1, \ldots, x_{s} .
$$

Proof. This is a consequence of Lemma 6.2 and Theorem 4.8.

The next application concerns results of Huckaba on the relation type of certain ideals. Following [10], we call a sequence $x_{1}, \ldots, x_{s}$ of elements of a local ring $A$ a complete $d$-sequence with respect to $I$ if $x_{1}, \ldots, x_{s}$ is a $d$-sequence and the initial forms of $x_{1}, \ldots, x_{s-1}$ form a regular sequence in $G=G_{I}(A)$. Notice that the definition in [10] is restricted only to the case $\ell(I)=$ ht $I+1$.

Example. Every minimal induction of $I$ is generated by a complete $d$-sequence with respect to $I$ in the following cases:

(1) grade $I=$ ht $I$ and $\ell(I)=$ ht $I \leq \operatorname{grade} G_{+}+1$.

(2) $A$ is a Cohen-Macaulay ring and $I$ is generically a complete intersection with $\ell(I)=$ ht $I+1=\operatorname{grade} G_{+}+1[10$, Proposition 1.2].

Huckaba proved that in the above cases, reltype $R_{I}(A) \leq r_{J}(I)+1$ for any minimal reduction $J$ of $I$ ([9, Theorem 2.3]; [10, Theorem 1.4]). We will improve his results by proving the relation $\operatorname{reg} R_{I}(A)=r_{J}(I)$. This yields an affirmative answer to his question of whether in case $(2)$ the reduction number $r_{J}(I)$ is independent of the choice of $J$; cf. [10, Theorem 1.7]. See also [23] and [24] for related results on this topic.

Theorem 6.4. Let $A$ be a local ring and $I$ an ideal of $A$. Let $J$ be a minimal reduction of $I$ which can be generated by a complete $d$-sequence with respect to $I$. Then

$$
\operatorname{reg} R_{I}(A)=r_{J}(I)
$$

Proof. Let $x_{1}, \ldots, x_{s}$ be a complete $d$-sequence with respect to $I$ which generates $J$, and let $r=r_{J}(I)$. By [27], the assumption that $x_{1}^{*}, \ldots, x_{s-1}^{*}$ is a regular sequence implies that $x_{1}, \ldots, x_{s-1}$ is a regular sequence and

$$
\left(x_{1}, \ldots, x_{i-1}\right) \cap I^{r+1}=\left(x_{1}, \ldots, x_{i-1}\right) I^{r}, \quad i=1, \ldots, s .
$$

From this it follows that for $i=1, \ldots, s-1$,

$$
\left[\left(x_{1}, \ldots, x_{i-1}\right): x_{i}\right] \cap I^{r+1}=\left(x_{1}, \ldots, x_{i-1}\right) I^{r} .
$$


Further, since $\left[\left(x_{1}, \ldots, x_{s-1}\right): x_{s}\right] \cap J=\left(x_{1}, \ldots, x_{s-1}\right)$ and $I^{r+1}=J I^{r}$, we also have

$$
\left[\left(x_{1}, \ldots, x_{s-1}\right): x_{s}\right] \cap I^{r+1}=\left(x_{1}, \ldots, x_{s-1}\right) \cap I^{r+1}=\left(x_{1}, \ldots, x_{s-1}\right) I^{r} .
$$

Therefore, using Theorem 4.8 we get $\operatorname{reg} R=r$.

The last application addresses the problem of describing ideals which yield Cohen-Macaulay blowing-ups. Given any positively graded ring $S$, we will denote by $\operatorname{Proj} S$ the set of all graded prime ideals not containing $S_{+}$. For an ideal $I$ of a ring $A, \operatorname{Proj} R_{I}(A)$ is called the blowing-up of $A$ along $I$.

Theorem 6.5. Let $(A, \mathfrak{m})$ be an equidimensional local ring which is a homomorphic image of a regular local ring with algebraically closed residue field. Let I be an $\mathfrak{m}$ primary ideal and $\operatorname{dim} A=s$. Then $\operatorname{Proj} R_{I}(A)$ is a Cohen-Macaulay scheme if and only if every minimal reduction $J=\left(x_{1}, \ldots, x_{s}\right)$ of $I$ satisfies the following condition for some $r \geq r_{J}(I)$ :

$$
\left[\left(x_{1}, \ldots, x_{i-1}\right): x_{i}\right] \cap I^{r+1}=\left(x_{1}, \ldots, x_{i-1}\right) I^{r}, \quad i=1, \ldots, s .
$$

Proof. $(\Rightarrow)$ By [26, Lemma 2.1 and Proposition 3.3] the assumption that Proj $R$ is a Cohen-Macaulay scheme implies that $\operatorname{Proj} G$ is a Cohen-Macaulay scheme, too. Since $x_{1}^{*}, \ldots, x_{s}^{*}$ form a homogeneous system of parameters for $G$, it follows that $x_{i}^{*} \notin P$ for any associated prime $P$ of $G /\left(x_{1}^{*}, \ldots, x_{i-1}^{*}\right), P \nsupseteq G_{+}, i=1, \ldots, s$. That means $x_{1}^{*}, \ldots, x_{s}^{*}$ is a filter-regular sequence of $G$. Thus, $x_{1}, \ldots, x_{s}$ satisfies the above condition by Theorem 4.8 .

$(\Leftarrow)$ Let $P \in \operatorname{Proj} R$ be maximal. Then $P_{0}=\mathfrak{m}$. Since $R / \mathfrak{m} R$ is a finitely generated algebra over the algebraically closed field $A / \mathfrak{m}$, there is a minimal basis $x_{1}, \ldots, x_{v}$ of $I$ such that $P$ is generated by $\mathfrak{m}$ and the set $\left\{x_{1} t, \ldots, x_{v} t\right\} \backslash\left\{x_{s}^{t}\right\}$. Let $R_{P}$ be the homogeneous localization of $R$ at $P$. Then $R_{P}$ is the localization of the ring $B:=A\left[x_{1} / x_{s}, \ldots, x_{v} / x_{s}\right]$ at the maximal ideal generated by $\mathfrak{m}$ and $\left\{x_{1} / x_{s}, \ldots, x_{v} / x_{s}\right\} \backslash\left\{x_{s} / x_{s}\right\}$. Without restriction we may assume that $J=\left(x_{1}, \ldots, x_{s}\right)$ is a minimal reduction of $I$. Then $x_{1}, \ldots, x_{s}$ satisfies the condition of Theorem 6.5. Hence $x_{1}^{*}, \ldots, x_{s}^{*}$ is a filter-regular sequence of $G$ by Theorem 4.8. By Lemma 3.6, $x_{1} t, \ldots, x_{s} t$ is a filter-regular sequence of $R$. It follows that $x_{i} t \notin Q$ for any associated prime ideal $Q$ of $R /\left(x_{1} t, \ldots, x_{i-1} t\right), x_{s} t \in Q$. Therefore, $x_{1} / x_{s}, \ldots, x_{s-1} / x_{s}$ is a regular sequence of $B$ which is the homogeneous localisation of $R$ with respect to the element $x_{s} t$. Now we will show that

$$
\left(x_{1} / x_{s}, \ldots, x_{s-1} / x_{s}\right) B: x_{s}=\left(x_{1} / x_{s}, \ldots, x_{s-1} / x_{s}\right) B .
$$

This relation of elements of $B$ can be translated as a relation of elements of $A$ :

$$
\left[\left(x_{1}, \ldots, x_{s-1}\right) I^{n-1}: x_{s}\right] \cap I^{n}=\left(x_{1}, \ldots, x_{s-1}\right) I^{n-1}
$$

for all large $n$. But as we have seen in the remark following Theorem 4.8, this condition is equivalent to the condition

$$
\left[\left(x_{1}, \ldots, x_{s-1}\right): x_{s}\right] \cap I^{n}=\left(x_{1}, \ldots, x_{s-1}\right) I^{n-1}
$$

which is satisfied for $n \geq r+1$ by Proposition 4.7 (i). Thus $x_{1} / x_{s}, \ldots, x_{s-1} / x_{s}, x_{s}$ is a regular sequence of $B$. Since $\operatorname{dim} B_{P}=h t P=s$, this implies that $B_{P}$ is a Cohen-Macaulay ring.

Remark. The author guesses that there is a similar characterization for Proj $R_{I}(A)$ to be a Cohen-Macaulay ring when $I$ is an ideal of $A$ with analytic spread $\ell(I)=$ $\operatorname{dim} A$. 
From Theorem 6.5 we immediately obtain the following interesting consequence.

Corollary 6.6. Let $(A, \mathfrak{m})$ be a local ring as in Theorem 6.5. Then the following conditions are equivalent:

(i) $\operatorname{Proj} R_{I}(A)$ is a Cohen-Macaulay scheme for every parameter ideal I of A.

(ii) Every system of parameters of $A$ is a sequence of regular type.

Remark. For an arbitrary local ring $A$, Goto [4] has proved that (i) is equivalent to the condition that $A / H_{\mathfrak{m}}^{0}(A)$ is a Buchsbaum ring. From this one can easily deduce (ii), because every system of parameters of a Buchsbaum local ring is a $d$-sequence.

\section{REFERENCES}

[1] I. M. Aberbach, C. Huneke, and Ngô Viêt Trung, Reduction numbers, Briançon-Skoda theorems and the depth of Rees rings, Compositio Math. 97 (1995), 403-434. MR 96g:13002

[2] D.G. Costa, Sequences of linear type, J. Algebra 94 (1985), 256-263. MR 86h:13010

[3] D. Eisenbud and S. Goto, Linear free resolutions and minimal multiplicity, J. Algebra 88 (1984), 89-133. MR 85f:13023

[4] S. Goto, Blowing-up of Buchsbaum rings, In: Commutative Algebra, London Math. Soc. Lecture Notes 72, Cambridge Univ. Press, 1982, 140-162. MR 84h:13032

[5] S. Goto, Y. Nakamura, and K. Nishida, Cohen-Macaulay graded rings associated to ideals, Amer. J. Math. 118 (1996), 1196-1213. CMP 97:04

[6] J. Herzog, A. Simis, and W. Vasconcelos, Approximation complexes of blowing-up rings I, J. Algebra 18 (1982), 466-493. MR 83h:13023

[7] J. Herzog, A. Simis, and W. Vasconcelos, Koszul cohomology and blowing-up rings, in: Commutative Algebra, Marcel Dekker, New York, 1983, 79-169. MR 84k:13015

[8] L.T. Hoa, Reduction numbers of equimultiple ideals, J. Pure Appl. Algebra 109 (1996), 111126.

[9] S. Huckaba, Reduction numbers for ideals of higher analytic spread, Math. Proc. Camb. Phil. Soc. 102 (1987), 49-57. MR 89b:13023

[10] S. Huckaba, On complete $d$-sequences and the defining ideals of Rees algebras, Math. Proc. Camb. Phil. Soc. 106 (1989), 445-458. MR 90m:13012

[11] C. Huneke, On the symmetric and Rees algebras of an ideal generated by a $d$-sequence, J. Algebra 62 (1980), 268-275. MR 81d:13016

[12] C. Huneke, Symbolic powers of prime ideals and special graded algebras, Commun. Algebra 9 (1981), 339-366. MR 83a:13011

[13] C. Huneke, Powers of ideals generated by weak $d$-sequences, J. Algebra 62 (1981), 471-509. MR 82k: 13003

[14] C. Huneke, The theory of $d$-sequences and powers of ideals, Adv. in Math. 46 (1982), 249-279. MR 84g:13021

[15] M. Johnson and B. Ulrich, Artin-Nagata properties and Cohen-Macaulay associated graded rings, Compositio Math. 108 (1996), 7-29. MR 97f:13006

[16] B. Johnston and D. Katz, Castelnuovo regularity and graded rings associated to an ideal, Proc. Amer. Math. Soc. 123 (1995), 727-734. MR 95d:13005

[17] M. Kühl, Thesis, University of Essen, 1981.

[18] T. Marley, The reduction number of an ideal and the local cohomology of the associated graded ring, Proc. Amer. Math. Soc. 117 (1993), 335-341. MR 93d:13029

[19] A. Ooishi, Castelnuovo's regularity of graded rings and modules, Hiroshima Math. J. 12 (1982), 627-644. MR 84m:13024

[20] A. Ooishi, Genera and arithmetic genera of commutative rings, Hiroshima Math. J. 17 (1987), 47-66. MR 89a:13025

[21] F. Planas-Vilanova, On the module of effective relations of a standard algebra, preprint.

[22] K.N. Raghavan, Powers of ideals generated by quadratic sequences, Trans. Amer. Math. Soc. 343 (1994), 727-747. MR 94i:13007

[23] P. Schenzel, Castelnuovo's index of regularity and reduction numbers, In: Topics in Algebra, Part II, Banach Center Publication 26, Warsaw, 1990, 201-208. MR 93g:13013

[24] Ngô Viêt Trung, Reduction exponent and bounds for the degree of the defining equations of graded rings, Proc. Amer. Math. Soc. 101 (1987), 229-235. MR 89i:13031 
[25] Ngô Viêt Trung, Reduction numbers, a-invariants, and Rees algebras of ideals having small analytic deviation, In: Commutative Algebra, World Scientific, 1994, 245-262. MR 97f:13007

[26] Ngô Viêt Trung and S. Ikeda, When is the Rees algebra Cohen-Macaulay? Comm. Algebra 17 (1989), 2893-2922. MR 91a:13008

[27] P. Valabrega and G. Valla, Form rings and regular sequences, Nagoya Math. J. 72 (1978), 93-101. MR 80d:14010

[28] G. Valla, On the symmetric and Rees algebras of an ideal, Manuscripta Math. 30 (1980), 239-255; 33 (1981), 59-61. MR 83b:14017; MR 83b:14018

[29] O. Zariski and P. Samuel, Commutative Algebra II, Springer, 1975. MR 52:10706

Institute of Mathematics, Box 631, Bò Hô, Hanoi, Vietnam

E-mail address: nvtrung@thevinh.ac.vn 